\title{
Methimazole plus levothyroxine for treating hyperthyroidism in children: a systematic review and meta-analysis
}

\author{
Xue Wu ${ }^{1 \#}$, Xia Qin ${ }^{2 \#}$, Yi Yao ${ }^{3}$ \\ ${ }^{1}$ Department of Pediatrics - Preventive Health Care, Renmin Hospital, Hubei University of Medicine, Shiyan, China; ${ }^{2}$ Department of Pediatrics, \\ Renmin Hospital, Hubei University of Medicine, Shiyan, China; ${ }^{3}$ Department of Endocrine, Renmin Hospital, Hubei University of Medicine, \\ Shiyan, China \\ Contributions: (I) Conception and design: X Wu, X Qin, Y Yao; (II) Administrative support: Y Yao; (III) Provision of study materials or patients: \\ X Wu, X Qin, Y Yao; (IV) Collection and assembly of data: X Wu, X Qin, Y Yao; (V) Data analysis and interpretation: X Wu, X Qin, Y Yao; (VI) \\ Manuscript writing: All authors; (VII) Final approval of manuscript: All authors. \\ \#These authors contributed equally to this work. \\ Correspondence to: Yi Yao. Department of Endocrine, Renmin Hospital, Hubei University of Medicine, Shiyan 442000, China. Email: 278764942@qq.com.
}

Background: Hyperthyroidism is a disease of excessive synthesis and secretion of thyroid hormones, and there is a lack of studies that have systematically evaluated the efficacy of the combination in treating hyperthyroidism. This study aimed to systematically evaluate the effectiveness and safety of methimazole combined with levothyroxine for treating hyperthyroidism in children.

Methods: We searched PubMed, CNKI, Wanfang Database, EMBASE, Web of Science, and other online electronic databases to find correlation studies of methimazole combined with levothyroxine in treating hyperthyroidism in children from 2010 to 2021. Meta-analysis was performed using Stata 16 software.

Results: Finally, 15 relevant articles were included comprising 1,718 pediatric patients. Meta-analysis results indicated that compared with methimazole alone (control group), the experimental group administered methimazole + levothyroxine had no evident difference in the level of thyroid-stimulating hormone [standardized mean difference $(\mathrm{SMD})=-0.34,95 \%$ confidence interval $(\mathrm{CI}):-1.02,0.35, \mathrm{P}=0.33$ ], but notably improved the efficacy of clinical treatment of hyperthyroidism in children [odds ratio (OR) $=5.77,95 \%$ CI: 2.62, 12.74, $\mathrm{P}<0.001]$. Meanwhile, the experimental group had lower adverse reaction rates $(\mathrm{OR}=0.28,95 \% \mathrm{CI}: 0.19,0.40, \mathrm{P}<0.001)$, free triiodothyronine (FT3) level $(\mathrm{SMD}=-0.85,95 \% \mathrm{CI}:-1.57$, 0.13, $\mathrm{P}=0.02$ ), free tetraiodothyronine (FT4) level (SMD $=-0.94,95 \% \mathrm{CI}:-1.59,-0.30, \mathrm{P}=0.004)$ and reduced thyroid volume $(\mathrm{SMD}=-1.3,95 \% \mathrm{CI}:-1.67,0.93, \mathrm{P}<0.001)$.

Discussion: Using methimazole + levothyroxine to treat hyperthyroidism in children can raise the levels of FT3 and FT4, reduce the thyroid volume, improve clinical efficacy, and lower the adverse reaction rate of patients.

Keywords: Hyperthyroidism; levothyroxine; meta-analysis; methimazole

Submitted Sep 30, 2021. Accepted for publication Jan 07, 2022.

doi: 10.21037/tp-21-497

View this article at: https://dx.doi.org/10.21037/tp-21-497

\section{Introduction}

Hyperthyroidism is a disease of excessive synthesis and secretion of thyroid hormones, inducing symptoms such as goiter, hot flashes of the skin and metabolic disorders (1).
Thyroid stimulating antibody binds competitively with thyroid stimulating hormone (TSH) to the TSH receptor (TSHR)-a subunit, activating the adenylate cyclase signaling system, leading to thyroid follicular epithelial hyperplasia and excess thyroid hormone production. It is a rare but 
severe disease with a high possibility of complications. The incidence of fetal or childhood disease is between $0.0025 \%$ and $0.025 \%(2,3)$. The onset of hyperthyroidism in children is insidious, because they are less self-aware, and its early symptoms and abnormal emotional expression of the child are easily overlooked, losing the opportunity for treatment in the early stage (4).

At present, there are three approaches to treating hyperthyroidism: antithyroid medications, subtotal or near-total thyroidectomy, and nuclide iodine therapy (5). Surgery is difficult with high risk, so for children, the first choice is drug therapy. Methimazole and levothyroxine are commonly used antithyroid drugs. Methimazole inhibits the synthesis of thyroid hormones at different stages in the thyroid and peripheral tissues to reduce the levels of thyroid hormones (6). Levothyroxine has a similar function to the human endogenous hormones. It is metabolized by deiodination and glucuronidation, thereby ameliorating symptoms (7). The treatment mechanisms and effects of the two drugs are completely different. Studies have shown that initial use of high-dose methimazole is harmful to children and adolescents (8), but long-term administration is an effective and safe treatment that also prevents recurrence of hyperthyroidism (9-11). The use of methimazole alone can easily cause secondary attenuation to thyroid function, which levothyroxine can ameliorate (12-14); therefore, their combination for long-term treatment may not only effectively treat the disease, but also reduce the incidence of functional attenuation. However, currently there is a lack of studies that have systematically evaluated the efficacy of the combination in treating hyperthyroidism, so we systematically analyzed the effectiveness and safety of methimazole combined with levothyroxine in treating hyperthyroidism in children through a meta-analysis. We present the following article in accordance with the PRISMA reporting checklist (available at https:// tp.amegroups.com/article/view/10.21037/tp-21-497/rc).

\section{Methods}

\section{Literature search}

We followed the guidelines of the systematic review and meta-analysis (PRISMA-P) protocol statement (15), using "methimazole", "treatment" and "hyperthyroidism" as keywords and Boolean logic, to search for relevant articles from 2010 to 2021 in PubMed, CNKI, Wanfang, EMBASE, and Web of Science, including dissertations, conference reports, and journal articles with no language restriction.

\section{Screening criteria}

Our inclusion criteria were as follows: (I) research subjects under 18 years of age and meeting the diagnostic criteria for hyperthyroidism, with no sex or nationality restrictions; (II) research type: randomized controlled trial (RCT) of drug treatment of hyperthyroidism, with no consideration of any blinding method; (III) intervention measures: experimental group administered methimazole + levothyroxine, and a control group treated with methimazole alone; (IV) outcome indicators: treatment efficacy and adverse reaction rate; levels of free triiodothyronine (FT3), free tetraiodothyronine (FT4), and thyroid-stimulating hormone (TSH), and thyroid volume before and after treatment in both the experimental and control groups.

Exclusion criteria: (I) case reports, news reports, and meta-analyses; (II) patients with other major diseases such as tumors; (III) studies with poorly defined processes and unclear conclusions.

\section{Data extraction}

Two researchers independently completed the literature search using NoteExpress 3.2 literature management software and determined whether the studies met the inclusion criteria based on the title, abstract, and methods. Then they evaluated the full text, and re-screened the literature based on the inclusion and exclusion criteria, and finally selected the articles for data extraction. The two researchers cross-checked relevant data and other materials. If there was any disagreement, they attempted to reach a consensus or consulted a third reviewer.

The data extraction details mainly included: (I) baseline information: name of the first author, publication year, and duration of the study; (II) subject information: duration of treatment, total number of subjects, age and sex of subjects; (III) experimental type: RCT; (IV) outcome indicators: treatment efficacy, adverse reaction rate, FT3, FT4, TSH, thyroid volume before and after treatment in both the experimental and control groups.

\section{Statistical analysis}

Meta-analysis was performed using Stata 16 software. Binary variables used odds ratio (OR) and $95 \%$ confidence interval (CI), standardized mean differences (SMDs) with the $95 \%$ CI were used as summary statistics for continuous variables. The $\mathrm{P}$ value was used to analyze and evaluate the heterogeneity between studies, and the Chi- 


\section{Identification of studies via databases and registers}

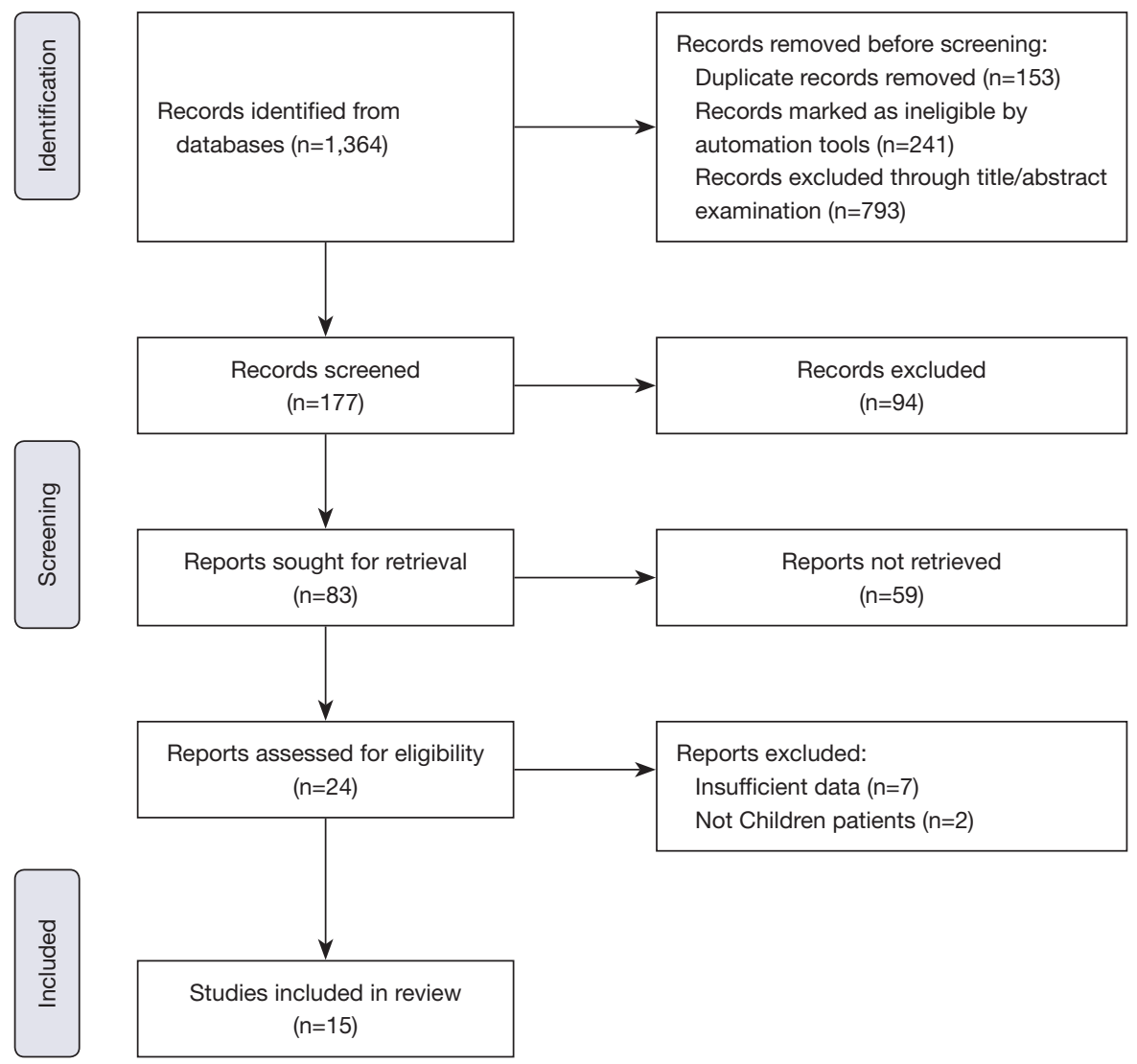

Figure 1 Flow chart of the literature search and screening process.

square test and $\mathrm{I}^{2}$ statistic were used to assess the extent of the heterogeneity. $\mathrm{P}<0.10$ and $\mathrm{I}^{2}>50 \%$ represented heterogeneity between the studies, and the randomeffects model was used for meta-analysis; otherwise, the fixed-effects model was selected. When the meta-analysis contained $\geq 10$, a funnel plot and Begg's test were used to judge the publication bias. Sensitivity analysis examined the stability of the overall results. $\mathrm{P}<0.05$ suggested a marked difference.

\section{Results}

\section{Literature search}

Using the described retrieval method, 1,364 related articles were initially retrieved, and 177 documents remained after excluding duplicate records. By reading the title and abstract, irrelevant studies were eliminated, and then the full text was examined according to the inclusion and exclusion criteria. Finally, 15 studies (16-30) were selected for this meta-analysis. Figure 1 shows the literature search and screening process.

\section{General situation of the included studies}

The 15 articles included 1,718 children diagnosed with hyperthyroidism. All studies were RCTs, of which 866 comprised the experimental (treatment) group and 852 were the control group. Treatment efficacy was the main outcome indicator. All 15 articles described the adverse reaction rate and FT3 and FT4 levels before and after treatment. There were 14 articles related to TSH before treatment and after treatment, and 12 articles recorded the thyroid volume before and after treatment. Table 1 shows the basic characteristics of the included studies. 


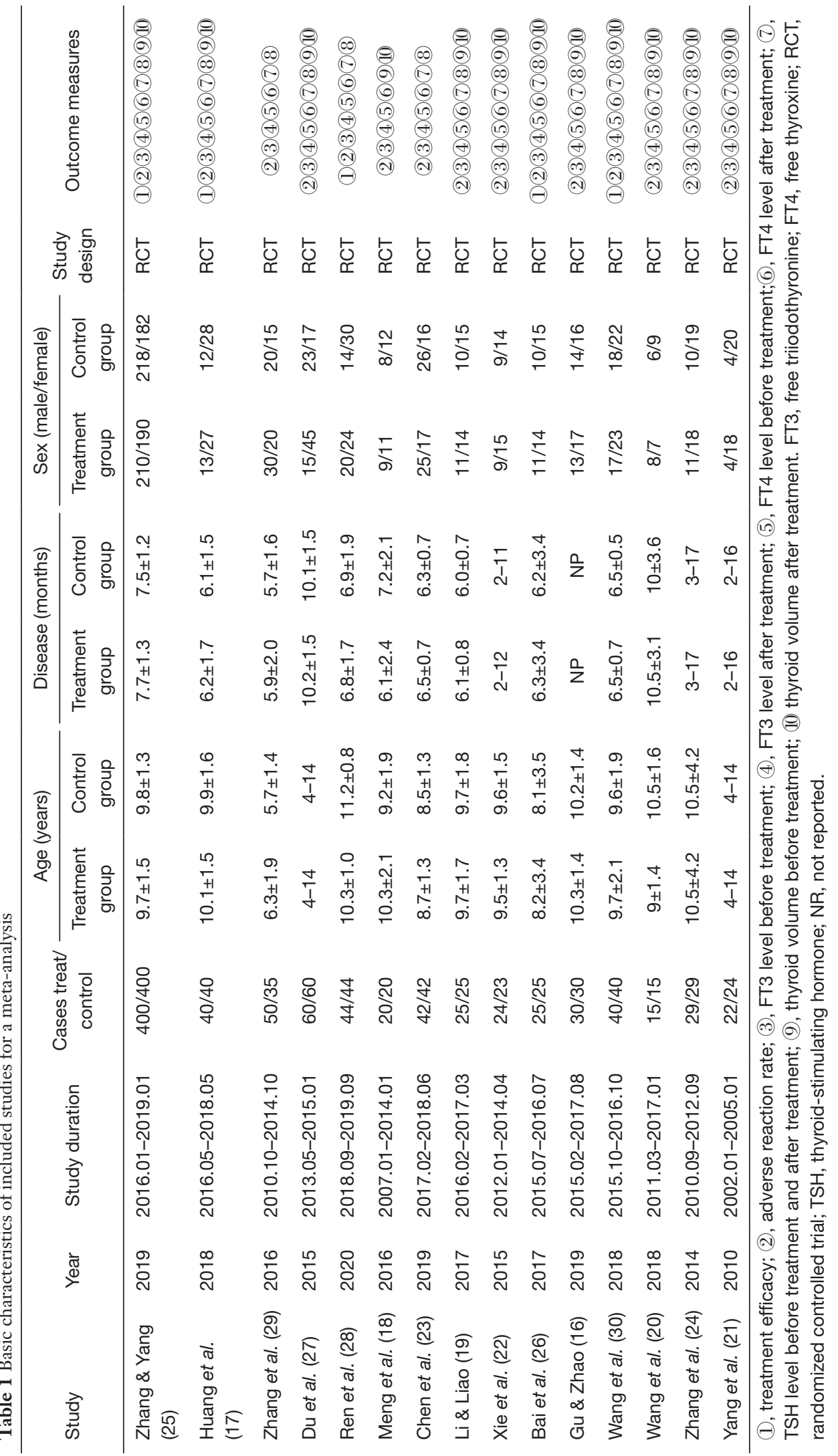




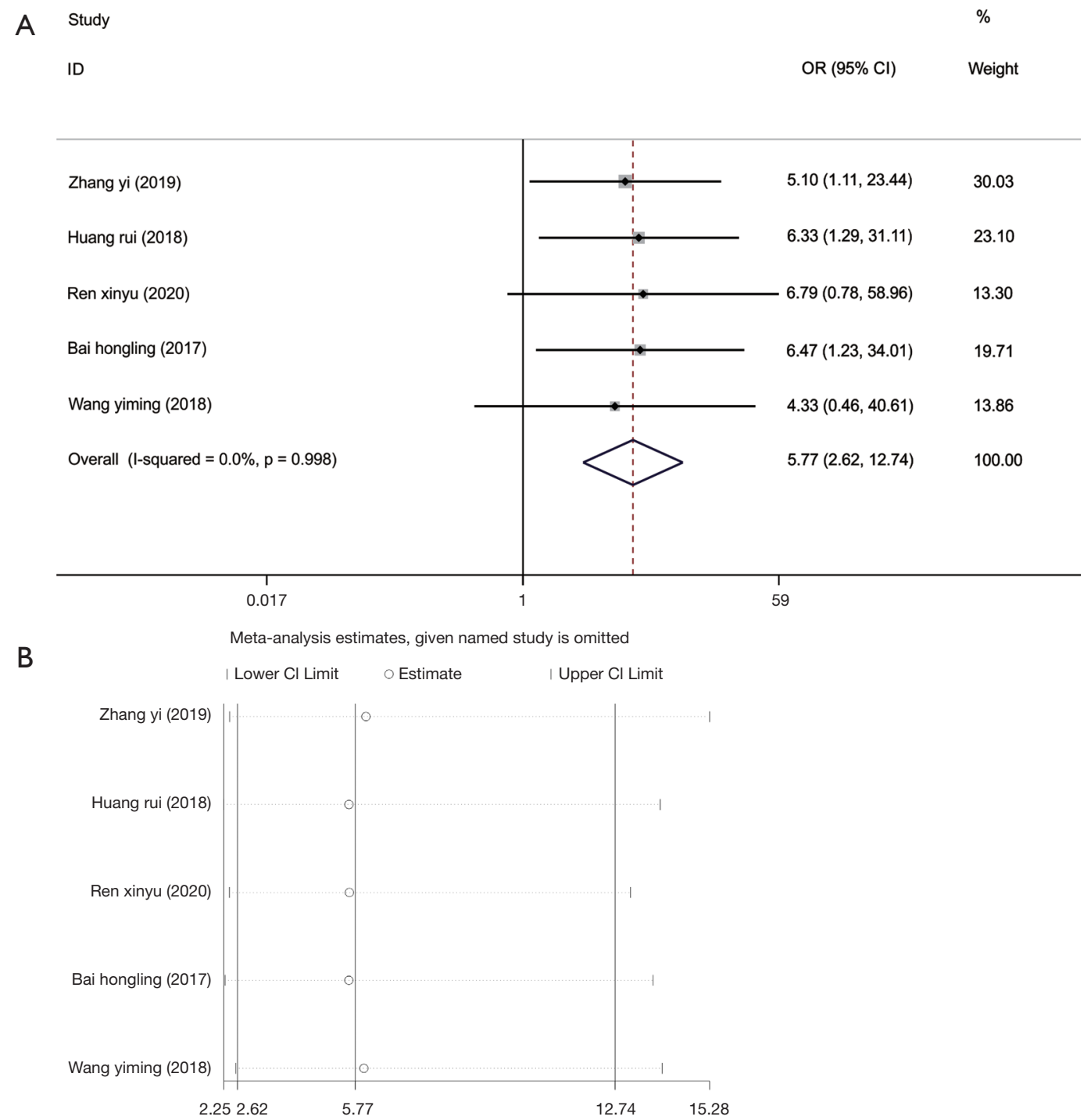

Figure 2 Meta-analysis of treatment efficacy for juvenile hyperthyroidism. (A) Forest chart and (B) sensitivity analysis of treatment efficacy. CI, confidence interval; OR, odds ratio.

\section{Treatment efficacy}

A total of five articles $(17,25,26,28,30)$ reported the efficacy of methimazole + levothyroxine in treating patients with hyperthyroidism. The included studies dis not have marked heterogeneity ( $\left.\mathrm{I}^{2}=0.0 \%, \mathrm{P}=0.998\right)$, so the fixed-effects model was selected for further analysis, and it showed that methimazole + levothyroxine was more effective than methimazole alone in treating hyperthyroidism in children $(\mathrm{OR}=5.77,95 \% \mathrm{CI}: 2.62,12.74, \mathrm{P}<0.001)$ (Figure $2 A)$. In order to re-estimate the pooled effect size, the research literature was eliminated one by one for sensitivity analysis, suggesting that the results before and after elimination did not change distinctly (Figure $2 B$ ), indicating low sensitivity and reliable and stable results.

\section{Adverse reaction rate}

All 15 studies (16-30) reported adverse reactions, but each 
had a different description of different adverse reactions. There was no distinct heterogeneity among the studies $\left(\mathrm{I}^{2}=8.3 \%, \mathrm{P}=0.36\right)$, so we chose a fixed-effects model for meta-analysis. The results revealed fewer adverse events in patients from the experimental group $(\mathrm{OR}=0.28,95 \% \mathrm{CI}$ : $0.19,0.40, \mathrm{P}<0.001$ ) (Figure $3 A$ ), indicating that treating with methimazole + levothyroxine was more universal than treating with methimazole alone. To explore the publication bias, a funnel plot was drawn based on the adverse reaction rate. All the studies were within the $95 \%$ CI except for one, and the plot was symmetrical. The P value of the Begg's test was 0.66 , suggesting no publication bias (Figure $3 B$ ). Sensitivity analysis of the adverse reaction rate revealed that the pooled effect size did not change evidently, so the results were stable (Figure 3C).

\section{Evaluation of FT3 and FT4 levels before and after treatment}

The level of FT3 is not affected by thyroid-binding globulin, and its reference level in the normal human body is $2.3-4.2 \mathrm{pg} / \mathrm{mL}$ (31). FT4 is a sensitive index for in vitro tests of thyroid function, which directly reflect thyroid function, and the level in patients with hyperthyroidism is generally $>23 \mathrm{pmol} / \mathrm{L}$ (32). Recording the levels of FT3 and FT4 before and after treatment is an evaluation of treatment efficacy. All 15 studies $(17-30,33)$ recorded the changes in FT3 and FT4 before and after treatment, with no distinct heterogeneity between the studies for FT3 and FT4 levels before treatment $\left(\mathrm{I}^{2}=0 \%, \mathrm{P}=0.999\right)$. The combined analysis results of the fixed-effect model showed that the experimental and control groups had no notable differences in FT3 or FT4 levels before treatment (FT3: SMD $=0.01$, 95\% CI: $-0.09,0.10, \mathrm{P}=0.869$; FT4: SMD $=0.01,95 \%$ CI: $-0.09,0.10, \mathrm{P}=0.872$ ) (Figure $4 A, 4 B$ ). However, after treatment, there was high heterogeneity between studies for the levels of FT3 and FT4, and the random-effects model was used to combine the effect size. From the results, both the FT3 (SMD $=-0.85,95 \% \mathrm{CI}:-1.57,0.13, \mathrm{P}=0.02$ ) and FT4 (SMD $=-0.94,95 \%$ CI: $-1.59,-0.30, \mathrm{P}=0.004)$ levels of the experimental group were markedly lower than those of the control group (Figure $4 C, 4 D$ ).

Publication bias analysis was performed by drawing the funnel plot of FT3 and FT4 before and after treatment, and the results indicated that all the effect values were within the 95\% CI (Figure 5), and the Begg's linear regression test results revealed that the study had no notable publication bias. The sensitivity analysis results of FT3 and FT4 levels before and after treatment all showed that the combined results of the study had low sensitivity and the results were stable (Figure 6).

\section{Evaluation of TSH level and thyroid volume before and after treatment}

There were 14 studies $(16,17,19-30)$ that measured the TSH levels in the plasma of patients before and after treatment, and 12 (16-22,24-27,30) compared the thyroid volume before and after treatment. The included studies showed no notable heterogeneity (thyroid volume: $\mathrm{I}^{2}=0 \%, \mathrm{P}=0.985$; TSH level: $\mathrm{I}^{2}=0 \%, \mathrm{P}=0.493$ ), so a fixed-effects model was selected for analysis, which revealed that the thyroid volume (SMD $=0.098,95 \% \mathrm{CI}:-0.004,0.20, \mathrm{P}=0.06)$ and the level of TSH in plasma (SMD $=0.07,95 \%$ CI: $-0.02,0.17$, $\mathrm{P}=0.13$ ) showed no evident differences between the two groups before treatment (Figure $7 A, 7 B$ ). After treatment, we selected a random-effects model for the analysis (thyroid volume: $\mathrm{I}^{2}=86.4 \%, \mathrm{P}=0.00$; TSH level: $\mathrm{I}^{2}=97.1 \%, \mathrm{P}=0.00$ ), and the results suggested that the thyroid volume of patients from the experimental group ( $\mathrm{SMD}=-1.3,95 \% \mathrm{CI}:-1.67$, $0.93, \mathrm{P}<0.001)$ was markedly lower than the control group, but there was no distinct difference in TSH level between the two groups (SMD $=-0.34,95 \% \mathrm{CI}:-1.02,0.35, \mathrm{P}=0.33$ ) (Figure $7 C, 7 D)$.

Publication bias analyses of thyroid volume and TSH level before and after treatment were conducted. The funnel plot results revealed that before and after treatment all the research points in the graph were concentrated within the $95 \% \mathrm{CI}$, with some symmetry and no publication bias (Figure $8 A, 8 B$ ). For thyroid volume before and after treatment, both the funnel plot and graph distributed asymmetrically before treatment, and $\mathrm{P}=0.015$ from the Begg's test (Figure 8C), indicating publication bias existed. However, the funnel plot of thyroid volume after treatment showed that all research points were distributed within the $95 \% \mathrm{CI}$, and $\mathrm{P}=0.061$, suggesting no publication bias (Figure 8D).

Sensitivity analyses of thyroid volume and TSH level before and after treatment demonstrated that the combined results of thyroid volume and TSH level before and after treatment had low sensitivity and were reliable and stable (Figure 9). 
A Study

ID

Zhang yi (2019)

Huang rui (2018)

Zhang zhiyu (2015)

Du mu (2015)

Ren xinyu (2020)

Meng yan (2016)

Chen wei (2019)

Li wenjin (2017)

Xie yiping (2015)

Bai hongling (2017)

Gu dongyun (2019)

Wang yiming (2018)

Wang lei (2018)

Zhang silu (2014)

Yang qin (2010)

Overall (I-squared $=8.3 \%, p=0.360$ )

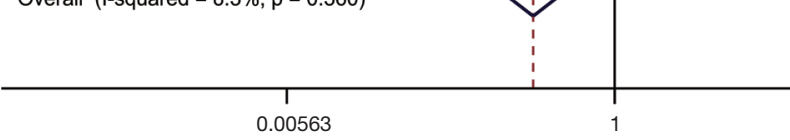

B

Begg's funnel plot with pseudo 95\% confidence limits

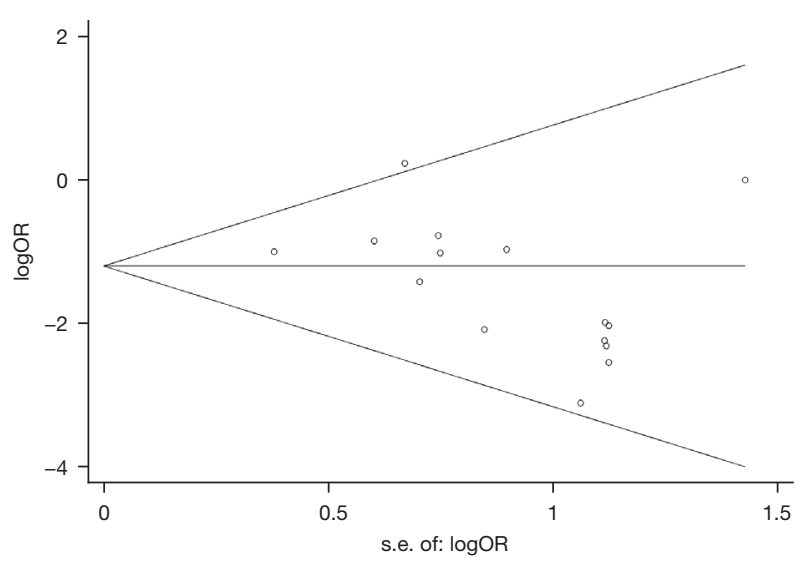

C

Meta-analysis estimates, given named study is omitted

| Lower Cl limit o Estimate | Upper Cl limit

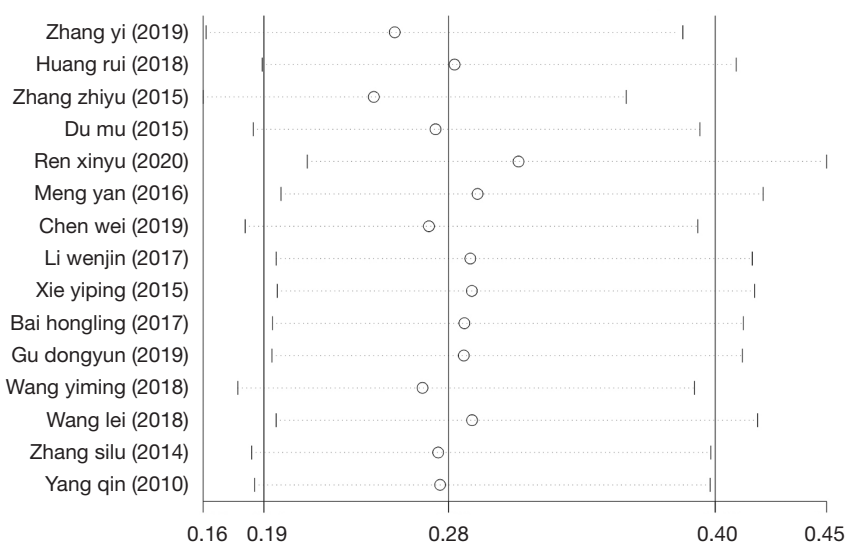

Figure 3 Meta-analysis of the adverse reaction rate after treatment of juvenile hyperthyroidism. (A) Forest diagram, (B) funnel plot and (C) sensitivity analysis of the adverse reaction rate. CI, confidence interval; OR, odds ratio. 


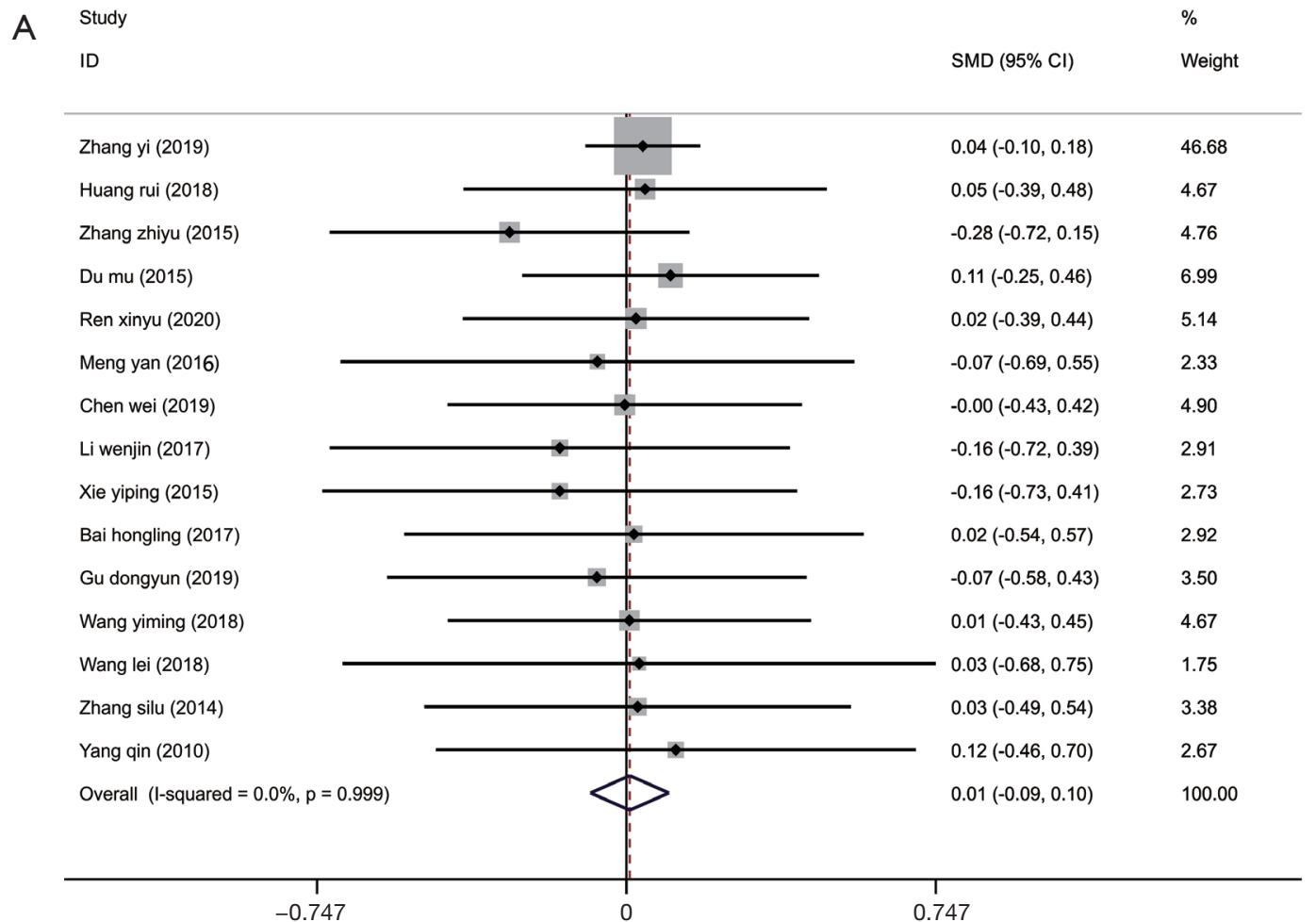

B Study
ID




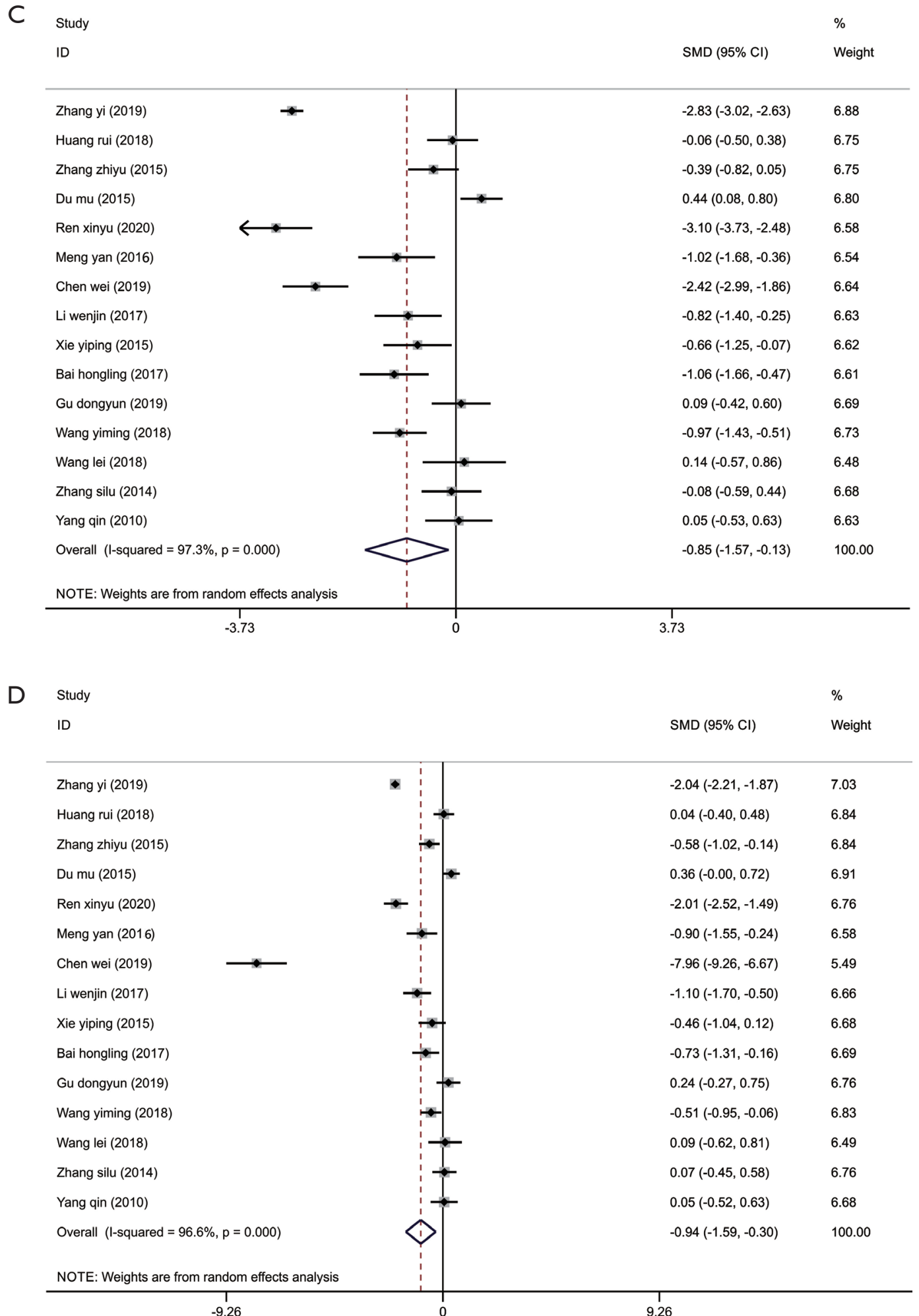

Figure 4 Forest plots of FT3 and FT4 levels before and after hyperthyroidism treatment. (A) FT3 levels before treatment; (B) FT4 levels before treatment; (C) FT3 levels before treatment; (D) FT4 levels after treatment. CI, confidence interval; FT3, free triiodothyronine; FT4, free thyroxine; SMD, standardized mean difference. 

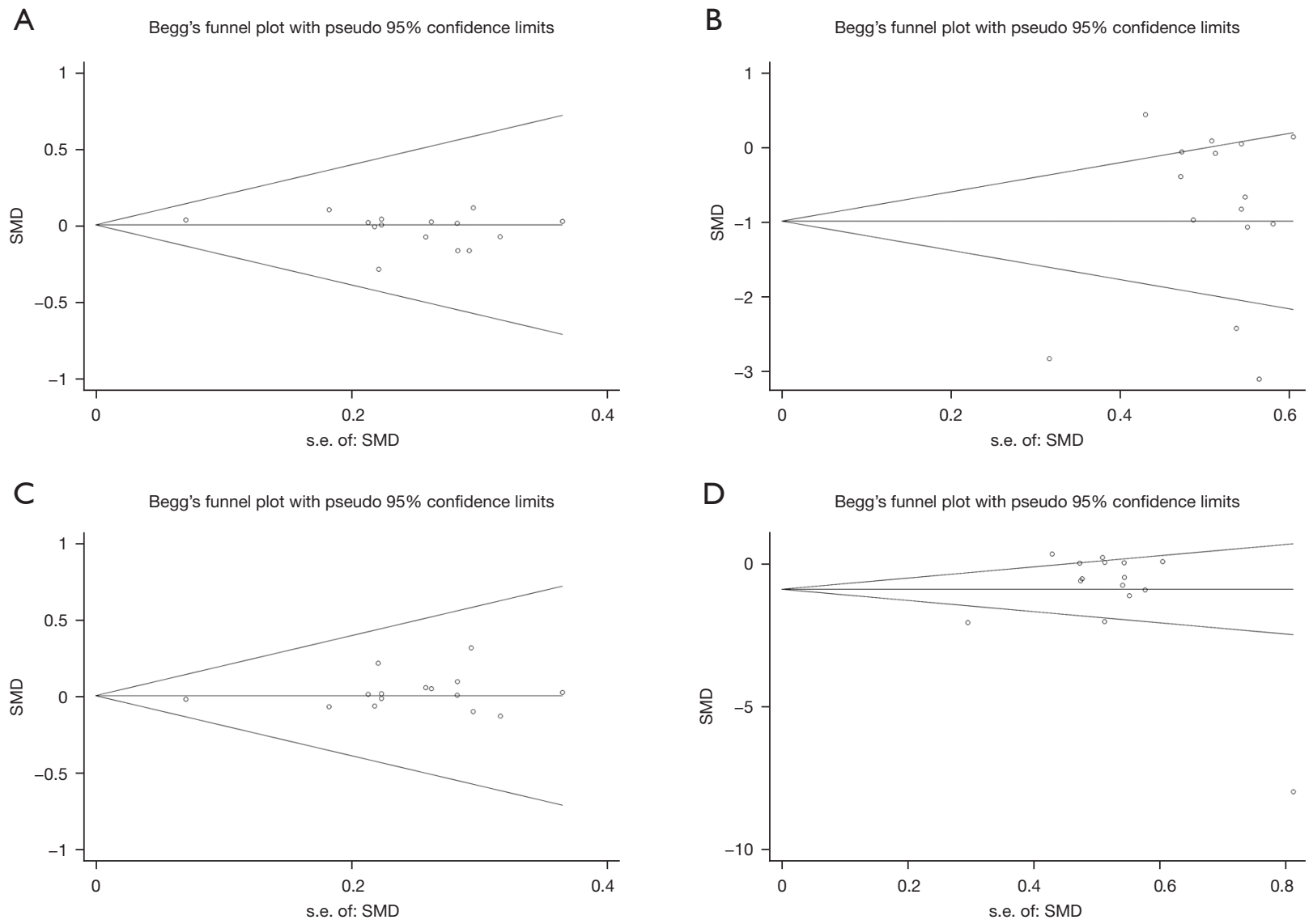

Figure 5 Publication bias funnel plots of biochemical indicators before and after hyperthyroidism treatment. FT3 levels (A) before and (B) after treatment. FT4 levels (C) before and (D) after treatment. FT3, free triiodothyronine; FT4, free thyroxine; SMD, standardized mean difference.

\section{Discussion}

Because children are growing and their body structure and tissues are not fully developed, the conservative method of drug treatment is mainly adopted for juvenile hyperthyroidism. During treatment, the main indicators of FT3, FT4, and TSH levels, and thyroid volume reflect the efficacy of treatment. The 2016 Japanese guidelines for hyperthyroidism in children recommended that antithyroid drugs should be administered for at least 18-24 months, and to use biochemical indicators to monitor disease (32). It has been reported that the TSH level in serum should be used as the initial screening and diagnostic criterion and that it has the highest sensitivity and specificity in assessing whether there is hyperthyroidism (34). However, others such as Bahn Chair et al. (35) have demonstrated that serum TSH level is still suppressed several months after taking antithyroid drugs, so it is not a good parameter for early detection and treatment. After continuous medication for 12-18 months, when the TSH level becomes normal, then reducing the dosage of methimazole or withdrawal can be considered (35). The TSH level is an effective indicator for evaluate treatment efficacy in juvenile hyperthyroidism. Long-term use of methimazole, an antithyroid drug, can reduce TSH levels and treat hyperthyroidism. Our study results indicated that methimazole + levothyroxine markedly reduced FT3 and FT4 levels and thyroid volume, but had no distinct effect on TSH levels, possibly because the average of the included studies was 7 months, which was shorter than is recommended in the treatment guidelines, 
A

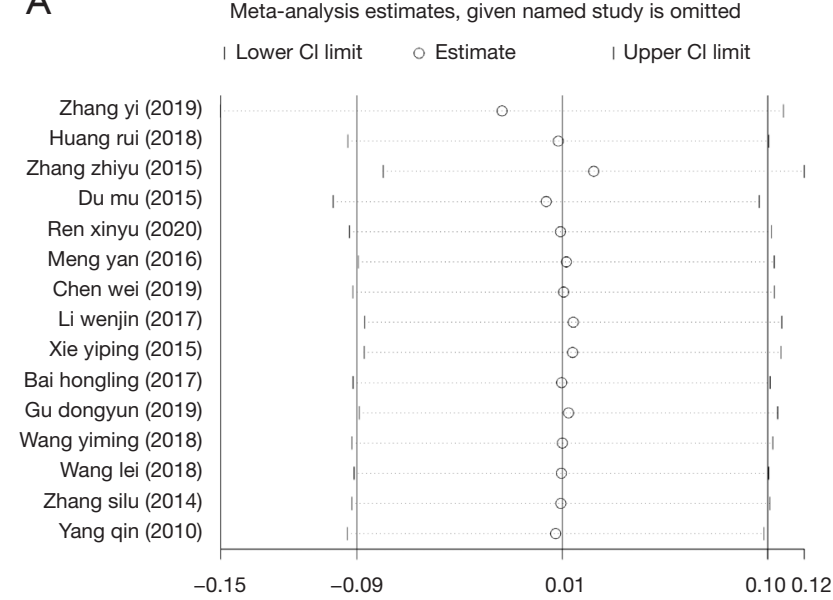

C

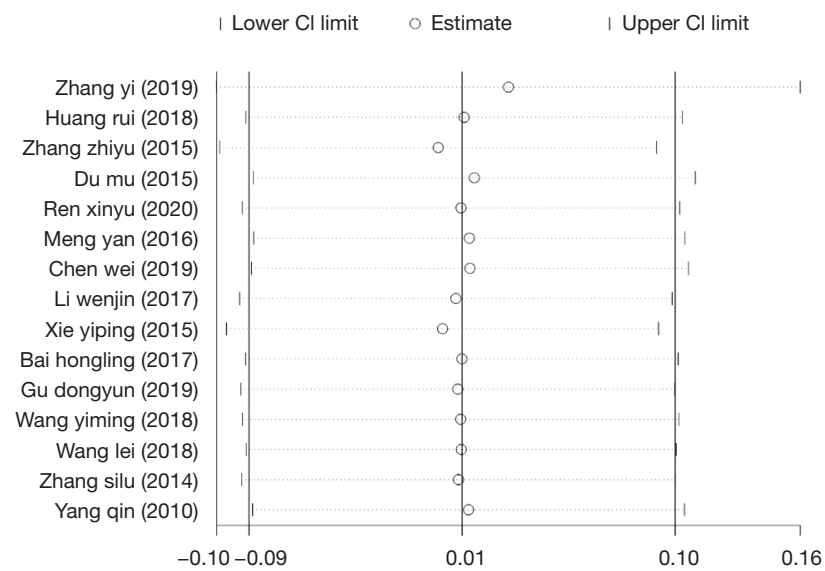

B

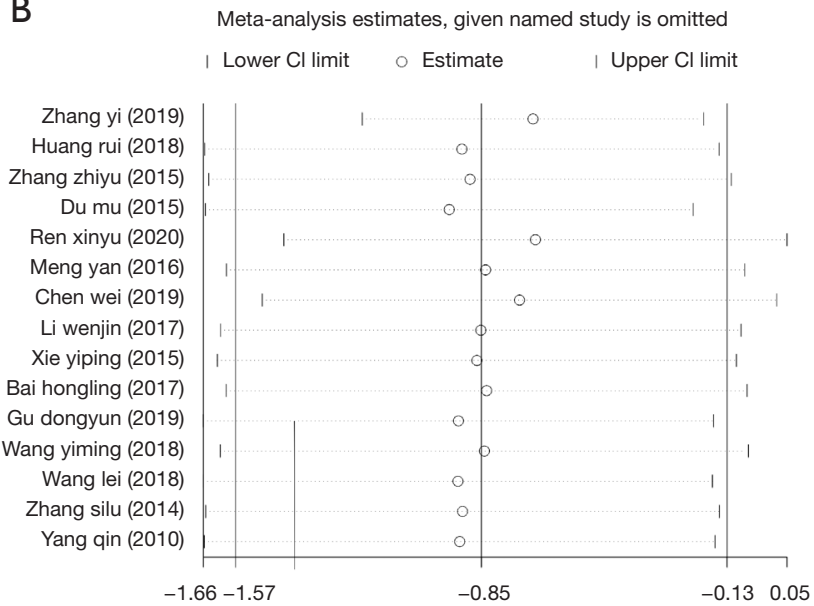

D

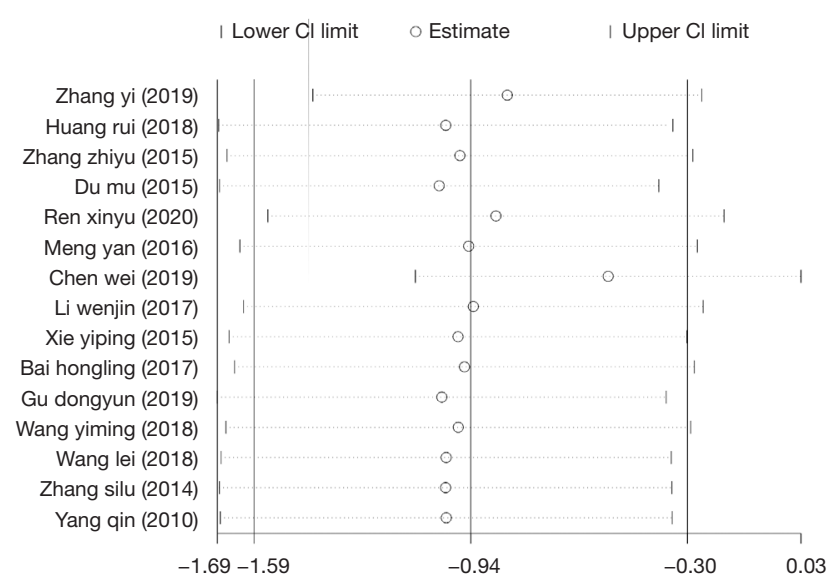

Figure 6 Sensitivity analyses of biochemical indicators before and after hyperthyroidism treatment. FT3 levels (A) before and (B) after treatment. FT4 levels (C) before and (D) after treatment. CI, confidence interval; FT3, free triiodothyronine; FT4, free thyroxine.

thereby affecting our conclusions.

Methimazole inhibits the synthesis and secretion of thyroid peroxidase, and can reduce the volume of the thyroid as well as the FT3 and FT4 levels and other indicators during the treatment of hyperthyroidism. When it is administered in different stages of the disease, the TSH level can increase, causing adverse reactions. However, the efficacy when using it alone in treating hyperthyroidism is not obvious, and its dosage and using combinations need to be further investigated clinically (36). Levothyroxine is a sodium salt of tetraiodothyronine, which decomposes to tetraiodothyronine after oral administration and strengthens the function of the sympathetic-adrenal system, so it is adjuvant therapy for hyperthyroidism with high biological activity and fast onset (37). As far as we know, current clinical treatment with the combination of these two drugs has a significant effect, but prognostic metaanalyses of children with hyperthyroidism are rare. Our meta-analysis found that compared with using methimazole alone, administering methimazole combined with levothyroxine had a better therapeutic effect on children with hyperthyroidism, with fewer adverse reactions, and effectively reduced the FT3 and FT4 levels, as well as the thyroid volume, providing a great reference for treating hyperthyroidism in children.

Although the 15 studies included in this study were 

ID

Zhang yi (2019)

Huang rui (2018)

Zhang zhiyu (2015)

Du mu (2015)

Ren xinyu (2020)

Chen wei (2019)

Li wenjin (2017)

Xie yiping (2015)

Bai hongling (2017)

Gu dongyun (2019)

Wang yiming (2018)

Wang lei (2018)

Zhang silu (2014)

Yang qin (2010)

Overall (I-squared $=0.0 \%, p=0.493$ )
A Study

$\%$

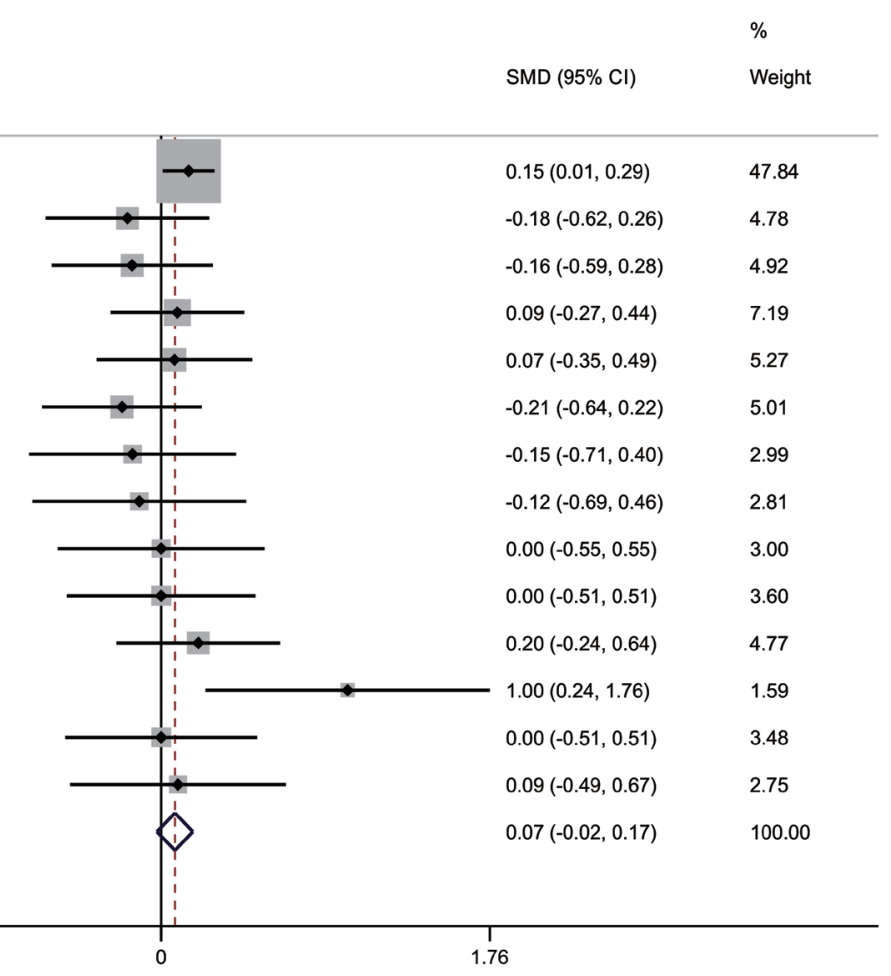

B Study

ID
$\%$

SMD $(95 \% \mathrm{Cl}) \quad$ Weight

\begin{tabular}{|c|c|c|c|}
\hline Zhang yi (2019) & $i$ & $0.17(0.03,0.31)$ & 54.69 \\
\hline Huang rui (2018) & $\frac{1}{4-1}$ & $0.06(-0.37,0.50)$ & 5.49 \\
\hline Du mu (2015) & & $-0.07(-0.42,0.29)$ & 8.23 \\
\hline Meng yan (2016) & & $-0.06(-0.68,0.56)$ & 2.74 \\
\hline Li wenjin (2017) & & $0.10(-0.45,0.66)$ & 3.43 \\
\hline Xie yiping (2015) & & $0.11(-0.46,0.69)$ & 3.22 \\
\hline Bai hongling (2017) & & $-0.02(-0.57,0.54)$ & 3.43 \\
\hline Gu dongyun (2019) & & $0.14(-0.36,0.65)$ & 4.11 \\
\hline Wang yiming (2018) & & $-0.01(-0.45,0.43)$ & 5.49 \\
\hline Wang lei (2018) & & $-0.07(-0.79,0.64)$ & 2.06 \\
\hline Zhang silu (2014) & & $-0.02(-0.54,0.49)$ & 3.98 \\
\hline Yang qin (2010) & & $-0.07(-0.64,0.51)$ & 3.15 \\
\hline Overall (I-squared $=0.0 \%, p=0.985$ ) & & $0.10(-0.00,0.20)$ & 100.00 \\
\hline
\end{tabular}




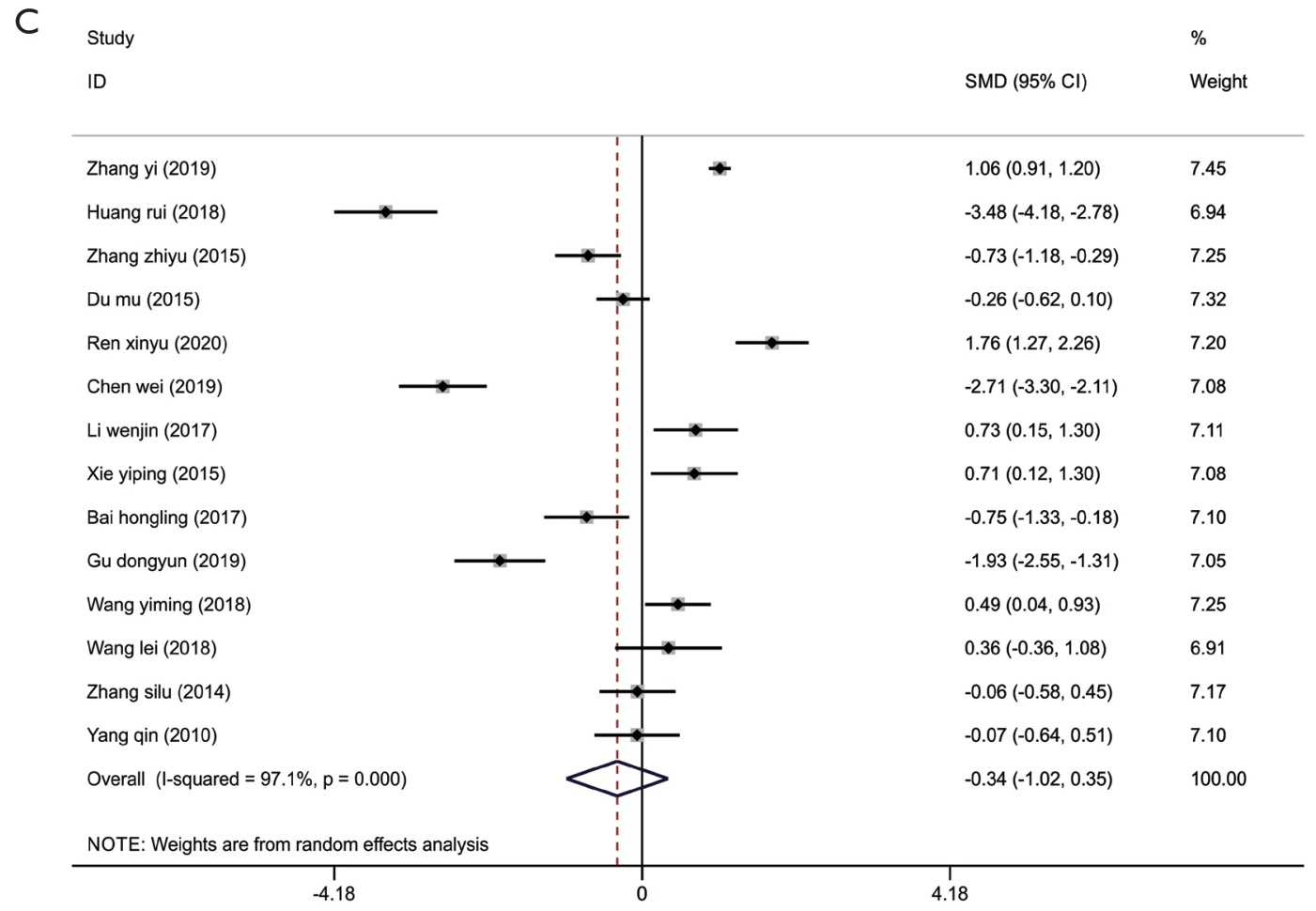

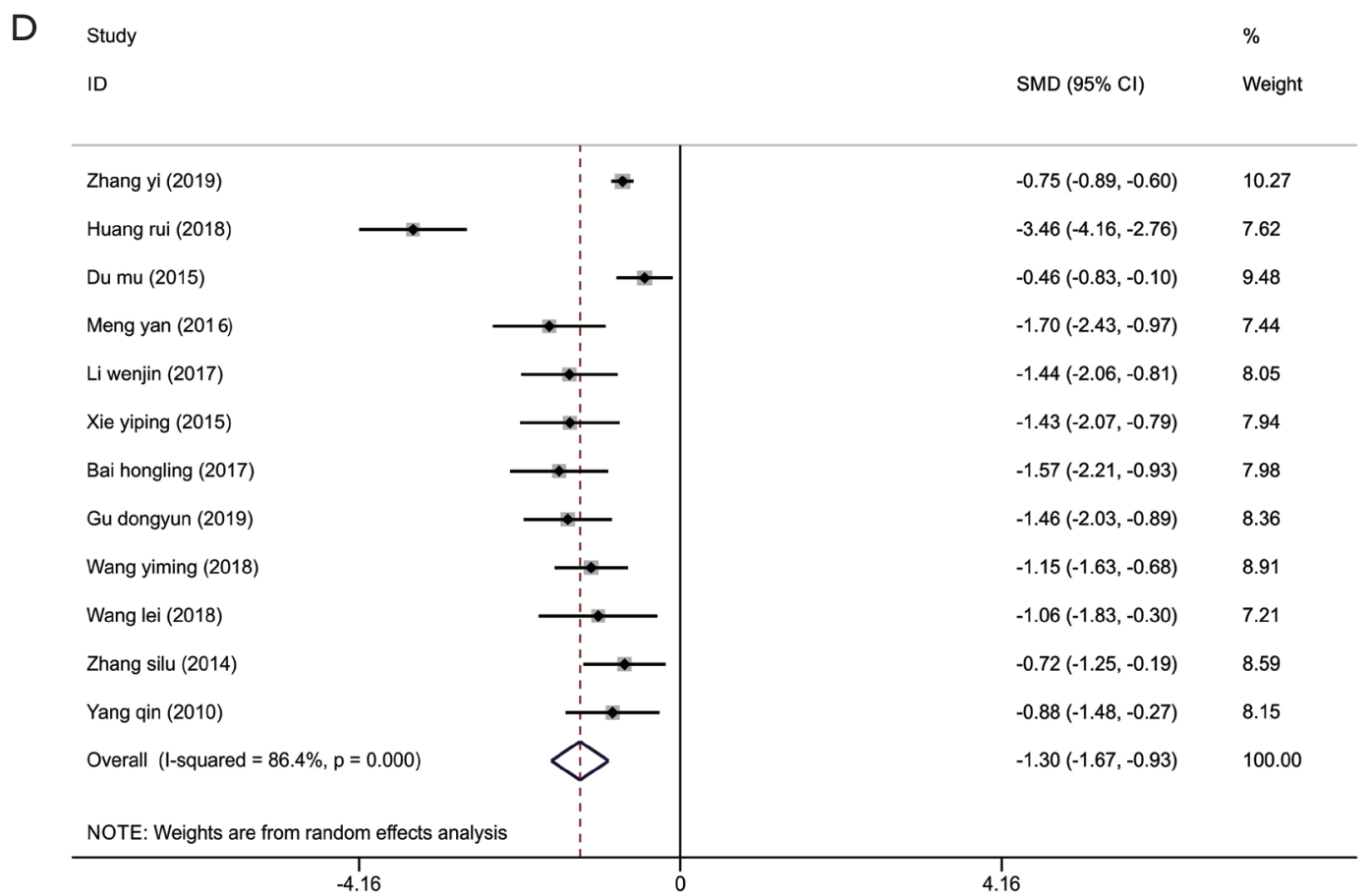

Figure 7 Forest plots of TSH levels and thyroid volume before and after hyperthyroidism treatment. (A) TSH levels before treatment; (B) thyroid volume before treatment; (C) TSH levels after treatment; (D) thyroid volume after treatment. TSH, thyroid-stimulating hormone; CI, confidence interval; SMD, standardized mean difference. 
A

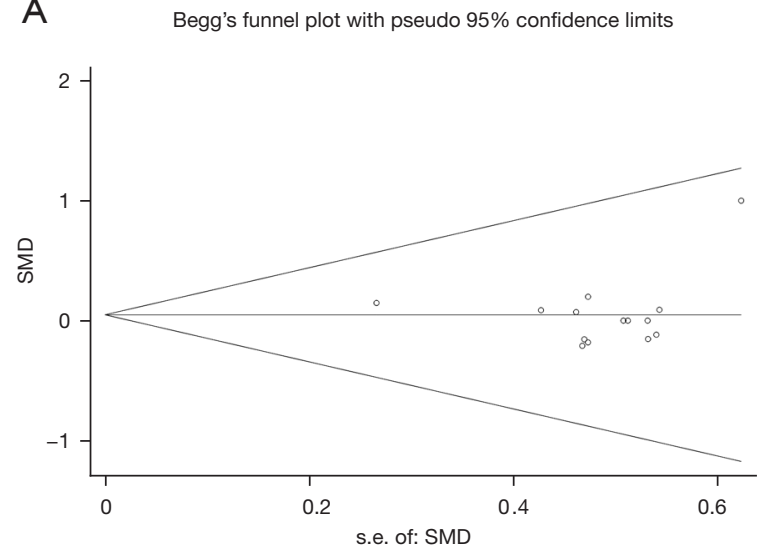

C

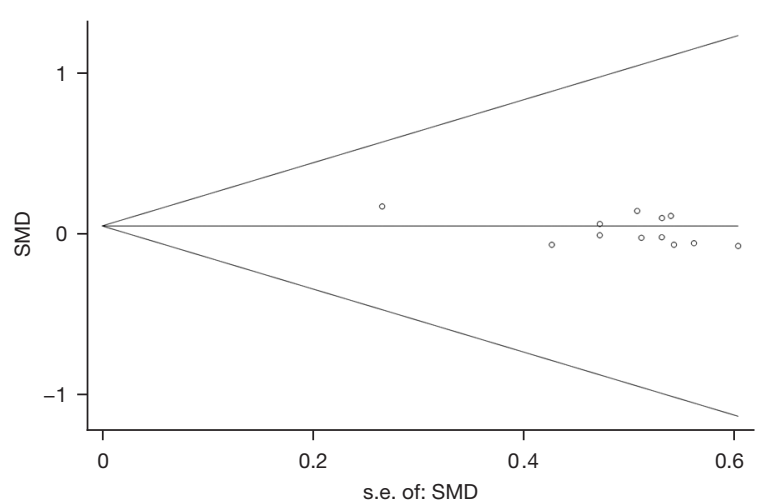

B Begg's funnel plot with pseudo $95 \%$ confidence limits

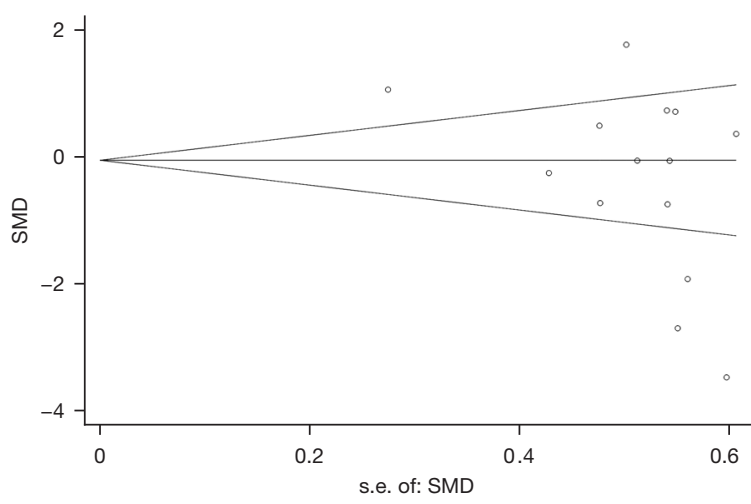

D Begg's funnel plot with pseudo $95 \%$ confidence limits

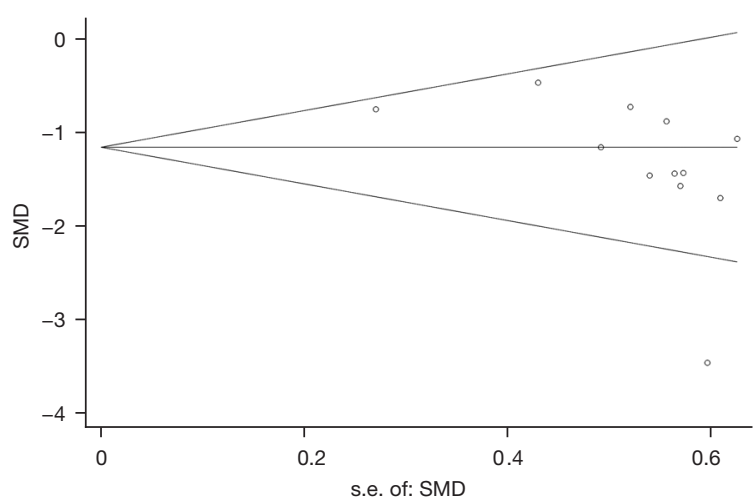

Figure 8 Begg's funnel plots of TSH levels and thyroid volume before and after hyperthyroidism treatment. Publication bias funnel plots of TSH (A) before and (B) after treatment. Publication bias funnel plots of thyroid volume (C) before and (D) after treatment. TSH, thyroidstimulating hormone; SMD, standardized mean difference.

all RCTs, with a total of 1,718 children included for heterogeneity, publication bias, and sensitivity, there are still some limitations. (I) The included 15 studies cannot not be guaranteed to have the same drug manufacturers, dosages, or treatment durations, so there will be clinical heterogeneity. (II) The studies failed to conduct in-depth research on the previous medical history of the children and other treatment-related factors (whether surgery and other drug treatments had been performed), and there may be side effects and sequelae that will affect treatment efficacy in this study. (III) There were only five studies reporting treatment efficacy, and as funnel plot analysis was not performed, publication bias is possible. (IV) The duration of drug administration was relatively short in all studies, none of which reached the treatment duration recommended by thyroid associations, which may affect the evaluation of the indicators.

\section{Conclusions}

Our meta-analysis found that methimazole combined with levothyroxine therapy can markedly improve the treatment efficacy of hyperthyroidism in children, and has advantages in decreasing FT3 levels, FT4 levels, thyroid volume, and the adverse reaction rate. However, this study still has the limitation of a small sample size, and the results require a larger sample and well-designed research for further verification. 
A

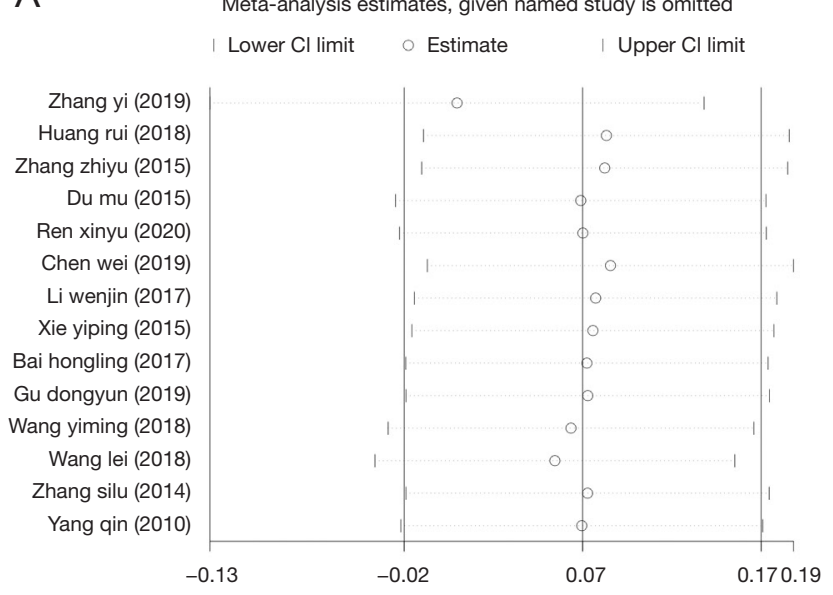

C

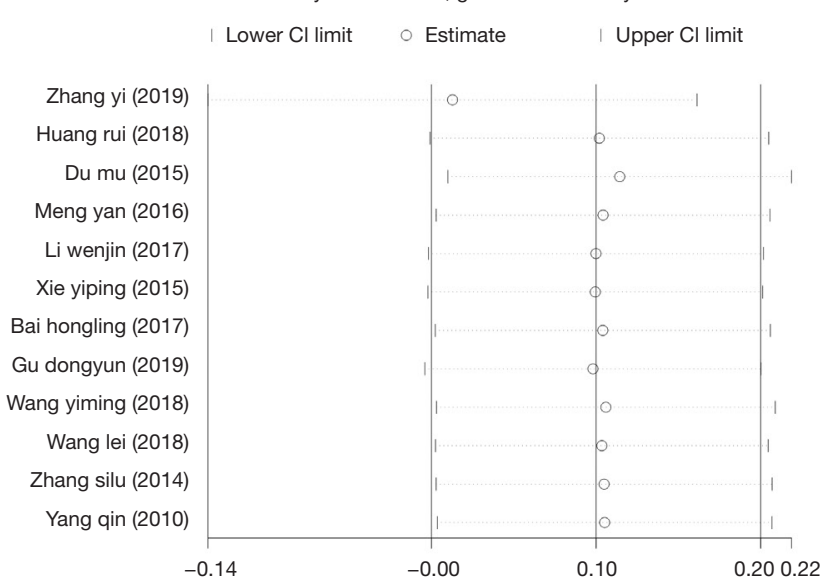

B

Meta-analysis estimates, given named study is omitted

| Lower Cl limit | Estimate Upper Cl limit

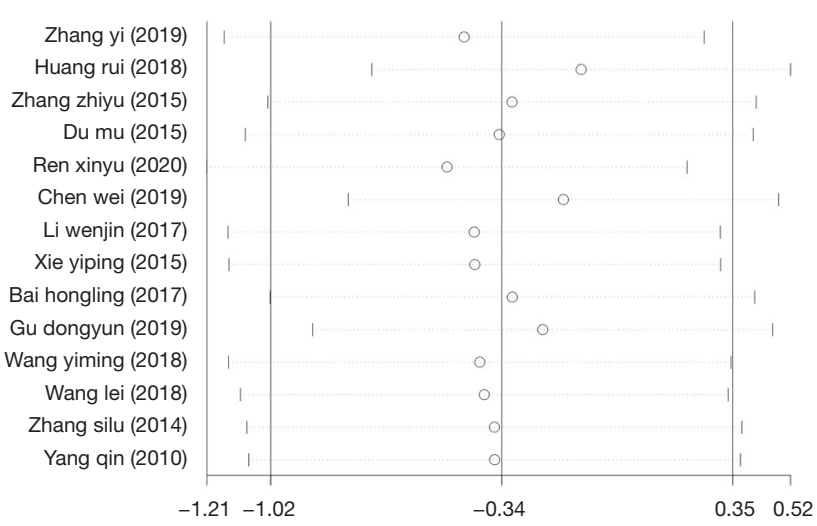

D

Meta-analysis estimates, given named study is omitted

| Lower Cl limit | Estimate Upper Cl limit

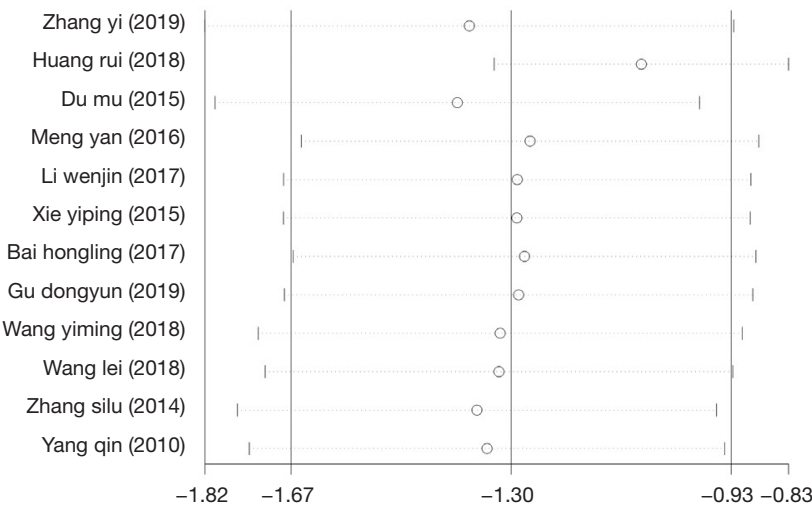

Figure 9 Sensitivity analysis of TSH levels and thyroid volume before and after hyperthyroidism treatment. Sensitivity analysis of TSH (A) before and (B) after treatment. Sensitivity analysis of thyroid volume (C) before and (D) after treatment. TSH, thyroid-stimulating hormone; SMD, standardized mean difference.

\section{Acknowledgments}

Funding: None.

\section{Footnote}

Reporting Checklist: The authors have completed the PRISMA reporting checklist. Available at https:// tp.amegroups.com/article/view/10.21037/tp-21-497/rc

Conflicts of Interest: All authors have completed the ICMJE uniform disclosure form (available at https://tp.amegroups. com/article/view/10.21037/tp-21-497/coif). The authors have no conflicts of interest to declare.

Ethical Statement: The authors are accountable for all aspects of the work in ensuring that questions related to the accuracy or integrity of any part of the work are appropriately investigated and resolved.

Open Access Statement: This is an Open Access article distributed in accordance with the Creative Commons Attribution-NonCommercial-NoDerivs 4.0 International License (CC BY-NC-ND 4.0), which permits the non- 
commercial replication and distribution of the article with the strict proviso that no changes or edits are made and the original work is properly cited (including links to both the formal publication through the relevant DOI and the license). See: https://creativecommons.org/licenses/by-nc-nd/4.0/.

\section{References}

1. De Leo S, Lee SY, Braverman LE. Hyperthyroidism. Lancet 2016;388:906-18.

2. Lavard L, Ranløv I, Perrild H, et al. Incidence of juvenile thyrotoxicosis in Denmark, 1982-1988. A nationwide study. Eur J Endocrinol 1994;130:565-8.

3. Radetti G, Zavallone A, Gentili L, et al. Foetal and neonatal thyroid disorders. Minerva Pediatr 2002;54:383-400.

4. Cui YM, Ruan ZW. Clinical Effect Analysis of Methimazole Combined with Levo-thyroxine in the Treatment of Hyperthyroidism in Children. Chinese and Foreign Medical Research 2019;17:45-6.

5. Léger J, Carel JC. Hyperthyroidism in childhood: causes, when and how to treat. J Clin Res Pediatr Endocrinol 2013;5 Suppl 1:50-6.

6. Fumarola A, Calvanese A, Di Fiore A, et al. Antithyroid drugs therapy. Clin Ter 2009;160:47-53.

7. Berger JL, Nguyen Y, Lebrun D, et al. Early neuropsychological adverse events after switching from $\mathrm{PI} / \mathrm{r}$ to dolutegravir could be related to hyperthyroidism in patients un-der levothyroxine. Antivir Ther 2017;22:271-2.

8. Sato H, Sasaki N, Minamitani K, et al. Higher dose of methimazole causes frequent adverse effects in the management of Graves' disease in children and adolescents. J Pediatr Endocrinol Metab 2012;25:863-7.

9. Azizi F, Abdi H, Amouzegar A. Control of Graves' hyperthyroidism with very long-term methimazole treatment: a clinical trial. BMC Endocr Disord 2021;21:16.

10. Azizi F, Ataie L, Hedayati M, et al. Effect of long-term continuous methimazole treatment of hyperthyroidism: comparison with radioiodine. Eur J Endocrinol 2005;152:695-701.

11. Bayramoğlu E, Elmaogulları S, Sagsak E, et al. Evaluation of long-term follow-up and methimazole therapy outcomes of pediatric Graves' disease: a single-center experience. J Pediatr Endocrinol Metab 2019;32:341-6.

12. Borson-Chazot F, Terra JL, Goichot B, et al. What Is the Quality of Life in Patients Treated with Levothyroxine for Hypothyroidism and How Are We Measuring It? A
Critical, Narrative Review. J Clin Med 2021;10:1386.

13. Hennessey JV. The emergence of levothyroxine as a treatment for hypothyroidism. Endocrine 2017;55:6-18.

14. Ho J, Jackson R, Johnson D. Massive levothyroxine ingestion in a pediatric patient: case report and discussion. CJEM 2011;13:165-8.

15. Moher D, Shamseer L, Clarke M, et al. Preferred reporting items for systematic review and meta-analysis protocols (PRISMA-P) 2015 statement. Syst Rev 2015;4:1.

16. Gu DY, Zhao YM. Efficacy of Metilimidazole Combined with Levothyroxine in Treatment of Hyperthyroidism in Children. Doctor 2019;4:104-5.

17. Huang R, Zhao HY, Chen SL, et al. Exploration on the curative effect and safety of methimazole combined with levothyroxine in treatment of children with hyperthyroidism. China Medicine and Pharmacy 2018;8:26-9.

18. Meng Y. The clinical effect of methimazole combined with levothyroxine in the treatment of hyperthyroidism in children. Jilin Medical Journal 2016;37:1327-8.

19. Li WJ, Liao B. Study on the effect of methimazole and levothyroxine in the treatment of children with hyperthyroidism. The World Clinical Medicine 2017;11:82.

20. Wang L. Analysis of Curative Effect of Methimidazole Combined with Levothyroxine in Treatment of Children with Hyperthyroidism. Systems Medicine 2018;3:133-5.

21. Yang Q. Observation of the curative effect of methimazole combined with levothyroxine in the treatment of hyperthyroidism in children. Contemporary Medicine 2010;16:9-10.

22. Xie YP, Luo JQ, Xie WX. Efficacy of methimazole combined with levothyroxine in the treatment of hyperthyroidism in children. Contemporary Medicine 2015;21:120-1.

23. Chen $W$. The clinical effect of methimazole combined with levothyroxine in the treatment of 42 children with hyperthyroidism. Journal of Clinical Research 2019;36:1609-11.

24. Zhang SL, Wei HY, Gu QR, et al. Clinical effect observation of methimazole combined with levothyroxine in the treatment of hyperthyroidism in children. China Modern Medicine 2014;21:64-6.

25. Zhang Y, Yang ZF. Evaluation of the effect of methimazole combined with levothyroxine in the treatment of children with hyperthyroidism. Modern Medicine and Health Research 2019;3:70-1.

26. Bai HL. Efficacy and adverse reactions of methimazole combined with levothyroxine in the treatment of 
children with hyperthyroidism. Chinese General Practice 2017;S2:173-5.

27. Du M. The clinical effect of methimazole combined with levothyroxine in the treatment of hyperthyroidism in children. Chinese Community Doctors 2015;31: 48, 50.

28. Ren XY. Evaluation of the effect of methimazole combined with levothyroxine in the treatment of children with hyperthyroidism. China Practical Medical 2020;15:146-8.

29. Zhang ZY. Clinical observation of methimazole combined with levothyroxine in the treatment of children with hyperthyroidism. China Practical Medical 2016;11:157-8.

30. Wang YM. Observation on the clinical effect of combined treatment of methimazole and levothyroxine in children with hyperthyroidism. Chinese and Foreign Medical Research 2018;16:122-3.

31. d'Herbomez M, Jarrige V, Darte C. Reference intervals for serum thyrotropin (TSH) and free thyroxine (FT4) in adults using the Access Immunoassay System. Clin Chem Lab Med 2005;43:102-5.

32. Committee on Pharmaceutical Affairs, Japanese Society for Pediatric Endocrinology, and the Pediatric Thyroid Disease Committee, Japan Thyroid Association (Taskforce for the Revision of the Guidelines for the Treatment of Childhood-Onset Graves' Dis-ease); Minamitani K, Sato $\mathrm{H}$, et al. Guidelines for the treatment of childhood-onset Graves' disease in Japan, 2016. Clin Pediatr Endocrinol

Cite this article as: $\mathrm{Wu} \mathrm{X}$, Qin X, Yao Y. Methimazole plus levothyroxine for treating hyperthyroidism in children: a systematic review and meta-analysis. Transl Pediatr 2022;11(1):41-57. doi: 10.21037/tp-21-497
2017;26:29-62.

33. Lu R, Wang H, Hong T, et al. Myopathy after rapid correction of hyperthyroidism: A case report and review of literature. Medicine (Baltimore) 2020;99:e18878.

34. de los Santos ET, Starich GH, Mazzaferri EL. Sensitivity, specificity, and cost-effectiveness of the sensitive thyrotropin assay in the diagnosis of thyroid disease in ambulatory patients. Arch Intern Med 1989;149:526-32.

35. Bahn Chair RS, Burch HB, Cooper DS, et al. Hyperthyroidism and other causes of thyrotoxicosis: management guidelines of the American Thyroid Association and American Association of Clinical Endocrinologists. Thyroid 2011;21:593-646. Erratum in: Thyroid 2012;22:1195.

36. Sato S, Noh JY, Sato S, et al. Comparison of efficacy and adverse effects between methimazole $15 \mathrm{mg}$ + inorganic iodine $38 \mathrm{mg} /$ day and methimazole $30 \mathrm{mg} /$ day as initial therapy for Graves' disease patients with moderate to severe hyperthyroidism. Thyroid 2015;25:43-50.

37. Tang ZF. Effect of Methimazole Combined with Levothyroxine on the Thyroid Volume and Serum FT3, FT4, TSH Levels of Patients with Hyperthyroidism. China \& Foreign Medical Treatment 2018;13:106-108.

(English Language Editor: K. Brown) 